ANNALES

POLONICI MATHEMATICI

$97.3(2010)$

\title{
The hyper-order of solutions of certain linear complex differential equations
}

\author{
by Guowei Zhang and Ang Chen (Jinan)
}

\begin{abstract}
We prove some theorems on the hyper-order of solutions of the equation $f^{(k)}-e^{Q} f=a\left(1-e^{Q}\right)$, where $Q$ is an entire function, which is a polynomial or not, and $a$ is an entire function whose order can be larger than 1 . We improve some results by J. Wang and X. M. Li.
\end{abstract}

1. Introduction and main results. We assume that the reader is familiar with the basic notions of Nevanlinna's value distribution theory (see [6, 9, 15, 17]). It will be convenient to let $E$ denote any set of positive real numbers of finite linear measure, not necessarily the same at each occurrence. For a nonconstant meromorphic function $f$, we denote by $T(r, f)$ the Nevanlinna characteristic of $f$ and by $S(r, f)$ any quantity satisfying $S(r, f)=o(T(r, f))(r \rightarrow \infty, r \notin E)$.

Let $f$ and $g$ be two nonconstant meromorphic functions and let $a$ be a complex number. We say that $f$ and $g$ share a $C M$ provided that $f-a$ and $g-a$ have the same zeros with the same multiplicities. Similarly, let $b \not \equiv \infty$ be a nonconstant meromorphic function such that $T(r, b)=S(r, f)$ and $T(r, b)=S(r, g)$. If $f-b$ and $g-b$ share $0 \mathrm{CM}$, we say that $f$ and $g$ share $b C M$. In this paper, we also need the following definitions.

Definition 1. For a nonconstant entire function $f$, the order $\sigma(f)$, lower order $\mu(f)$, hyper-order $\sigma_{2}(f)$ and lower hyper-order $\mu_{2}(f)$ are defined by

$$
\begin{aligned}
& \sigma(f)=\limsup _{r \rightarrow \infty} \frac{\log T(r, f)}{\log r}=\limsup _{r \rightarrow \infty} \frac{\log \log M(r, f)}{\log r}, \\
& \mu(f)=\liminf _{r \rightarrow \infty} \frac{\log T(r, f)}{\log r}=\liminf _{r \rightarrow \infty} \frac{\log \log M(r, f)}{\log r},
\end{aligned}
$$

2010 Mathematics Subject Classification: Primary 30D35.

Key words and phrases: hyper-order, entire function, linear complex differential equation, shared values. 


$$
\begin{aligned}
& \sigma_{2}(f)=\limsup _{r \rightarrow \infty} \frac{\log \log T(r, f)}{\log r}=\limsup _{r \rightarrow \infty} \frac{\log \log \log M(r, f)}{\log r}, \\
& \mu_{2}(f)=\liminf _{r \rightarrow \infty} \frac{\log \log T(r, f)}{\log r}=\liminf _{r \rightarrow \infty} \frac{\log \log \log M(r, f)}{\log r},
\end{aligned}
$$

respectively. Here and in what follows, $M(r, f)=\max _{|z|=r}|f(z)|$.

In 1977, L. A. Rubel and C. C. Yang [12] proved that if an entire function $f$ shares two distinct complex numbers CM with its derivative $f^{\prime}$, then $f=f^{\prime}$. What is the relation between $f$ and $f^{\prime}$ if the entire function $f$ shares one complex number $a \mathrm{CM}$ with its derivative $f^{\prime}$ ? In 1996, R. Brück [2] made a conjecture that if $f$ is a nonconstant entire function satisfying $\sigma_{2}(f)<\infty$, where $\sigma_{2}(f)$ is not a positive integer, and if $f$ and $f^{\prime}$ share one complex number $a \mathrm{CM}$, then $f-a=c\left(f^{\prime}-a\right)$ for some constant $c \neq 0$. In [2], R. Brück proved this conjecture for $a=0$, and also for $a \neq 0$ and $N\left(r, 1 / f^{\prime}\right)=S(r, f)$. In 1998, G. G. Gundersen and L. Z. Yang [5] proved that the conjecture is true for $a \neq 0$, provided that $\sigma(f)<\infty$. In 1999, L. Z. Yang [16] proved that if a nonconstant entire function $f$ and one of its derivatives $f^{(k)}$ share one complex number $a(\neq 0) \mathrm{CM}$, where $\sigma(f)<\infty$ and $k$ is a positive integer, then $f-a=c\left(f^{(k)}-a\right)$ for some complex number $c \neq 0$. In 2004, J. P. Wang proved the following theorem.

TheOrem A (see [14]). Let $f$ be a nonconstant entire function of finite order, let $P$ be a polynomial of degree $p \geq 1$, and let $k$ be a positive integer. If $f-P$ and $f^{(k)}-P$ share $0 C M$, then $f^{(k)}-P=c(f-P)$ for some complex number $c \neq 0$.

Regarding Theorem A, it is natural to ask what can be said if the order of $f$ is infinite. In [10], X. M. Li and C. C. Gao got the following result.

Theorem B (see [10]). Let $Q_{1}$ and $Q_{2}$ be two nonzero polynomials, and let $P$ be a polynomial. If $f$ is a nonconstant solution of the equation

$$
f^{(k)}-Q_{1}=e^{P}\left(f-Q_{2}\right),
$$

then $\sigma_{2}(f)=\operatorname{deg} P$.

Regarding Theorem B, what can be said if a nonconstant entire function $f$ and one of its derivative $f^{(k)}$ share an entire function $a$ which is a small function of $f$ ? In [13, J. Wang and X. M. Li proved the following theorem.

THEOREM $\mathrm{C}$ (see [13]). If $f$ is a nonconstant solution of the differential equation $f^{(k)}-a_{1}=\left(f-a_{2}\right) e^{Q}$, where $a_{1}$ and $a_{2}$ are two entire functions such that $\sigma\left(a_{j}\right)<1(j=1,2), k$ is a positive integer, and $Q$ is a polynomial, then $\mu_{2}(f)=\sigma_{2}(f)=\operatorname{deg} Q$.

From Theorem C, we know that the order of $a_{j}(j=1,2)$ must be less than 1 . What can be said if the order of $a_{j}$ is not less than 1 under 
the hypothesis of Theorem C? In this paper, we prove the following theorem.

THEOREM 1. If $f$ is a nonconstant solution of the differential equation

$$
f^{(k)}-a=(f-a) e^{Q},
$$

where $a$ is an entire function, $Q$ is a polynomial with $\operatorname{deg} Q<\sigma(a)<\infty$ and $k$ is a positive integer, then $\mu_{2}(f)=\sigma_{2}(f)=\operatorname{deg} Q$.

REMARK 1. From the proof of Theorem 1, we will see that if $Q$ is a constant, then $\operatorname{deg} Q=0$, thus, $\infty>\sigma(a)>0$; if $Q$ is a nonconstant polynomial, then $\operatorname{deg} Q \geq 1$, thus, $\infty>\sigma(a)>1$.

From Theorem 1 we get the following corollary which improves Theorem 1 of $[5]$.

COROLlARY 1. If $f$ is a nonconstant solution of the differential equation (1.1), where $a$ is an entire function, $Q$ is a nonconstant polynomial with $\operatorname{deg} Q<\sigma(a)<\infty$ and $k$ is a positive integer, then $\mu_{2}(f)=\sigma_{2}(f)=$ $\operatorname{deg} Q \geq 1$, and $f$ is an entire function of infinite order.

From Theorem 1 we also get the following two corollaries which improve Theorem A.

COROLlary 2. Let $f$ be a nonconstant solution of the differential equation (1.1), where $a$ is an entire function, $Q$ is a polynomial with $\infty>\sigma(a)>$ $\operatorname{deg} Q$ and $k$ is a positive integer. If $\mu_{2}(f)<\infty$ and $\mu_{2}(f)$ is not a positive integer, then $f^{(k)}-a=c(f-a)$ for some complex number $c \neq 0$.

COROLlary 3. Let $f$ be a nonconstant solution of the differential equation (1.1), where $a$ is an entire function, $Q$ is a polynomial with $\infty>\sigma(a)>$ $\operatorname{deg} Q$ and $k$ is a positive integer. If $\mu(f)<\infty$, then $f^{(k)}-a=c(f-a)$ for some complex number $c \neq 0$.

In Theorem $1, Q(z)$ is assumed to be a polynomial. What can be said if $Q(z)$ is a transcendental entire function? In [11, the authors proved the following theorem, assuming that $f$ satisfies a certain additional condition and $a=z$.

THEOREM D (see [11]). Let $Q$ be a transcendental entire function and $k$ be a positive integer. If $f$ is a solution of the equation

$$
\frac{f^{(k)}-z}{f-z}=e^{Q}
$$

and there exists a positive integer $l(2 \leq l \leq k)$ such that $m\left(r, 1 / f^{(l)}\right)=$ $O\{\log r T(r, f)\}(r \rightarrow \infty, r \notin E)$, where $E$ is a set of finite linear measure, then $\sigma_{2}(f)=\infty$. 
We continue this study using the method of [2] and get the following theorem, assuming that $\sigma(Q)<1 / 2$.

THeOREM 2. Let $Q$ be a transcendental entire function with $\sigma(Q)<1 / 2$, $a$ be an entire function of finite order and $k$ be a positive integer. If $f$ is a solution of the equation

$$
\frac{f^{(k)}-a}{f-a}=e^{Q}
$$

then $\sigma_{2}(f)=\infty$.

From Theorem 2 we get the following corollary.

COROLlary 4. Let $Q$ be a transcendental entire function with $\sigma(Q)<1 / 2$ and $k$ be a positive integer. If $f$ is a solution of the equation

$$
\frac{f^{(k)}-z}{f-z}=e^{Q}
$$

then $\sigma_{2}(f)=\infty$.

Comparing Theorem D with Corollary 4 suggests asking about the relationship between the condition $m\left(r, 1 / f^{(l)}\right)=O(\log r T(r, f))(r \rightarrow \infty$, $r \notin E$ ) (in Theorem D) and the condition $\sigma(Q)<1 / 2$ (in Corollary 4). It is an interesting question for further study.

2. Lemmas. Let $f(z)=\sum_{n=0}^{\infty} a_{n} z^{n}$ be an entire function. We define $\mu(r)=\max \left\{\left|a_{n}\right| r^{n}: n=0,1,2, \ldots\right\}$ and set $\nu(r, f)=\max \{m: \mu(r)=$ $\left.\left|a_{m}\right| r^{m}\right\}$, the central index of $f$ (see [5]).

Lemma 1 (see [9]). Let $g:(0, \infty) \rightarrow \mathbb{R}$ and $h:(0, \infty) \rightarrow \mathbb{R}$ be increasing functions such that $g(r) \leq h(r)$ outside an exceptional set $E$ of finite linear measure. Then, for any $\alpha>1$, there exists $r_{0}>0$ such that $g(r) \leq h(\alpha r)$ for all $r>r_{0}$.

Lemma 2 (see [8]). If $f$ is an entire function, then

$$
\sigma(f)=\limsup _{r \rightarrow \infty} \frac{\log \nu(r, f)}{\log r} .
$$

Lemma 3 (see [3]). If $f$ is a transcendental entire function, then

$$
\sigma_{2}(f)=\limsup _{r \rightarrow \infty} \frac{\log \log \nu(r, f)}{\log r} .
$$

LEMma 4 (see [13]). If $f$ is an entire function of infinite order, then

$$
\mu_{2}(f)=\liminf _{r \rightarrow \infty} \frac{\log \log \nu(r, f)}{\log r} .
$$


Lemma 5 (see [9]) Suppose that all the coefficients $a_{0}(\not \equiv 0), a_{1}, \ldots, a_{n-1}$ and $g(\not \equiv 0)$ of the nonhomogeneous linear differential equation

$$
f^{(n)}+a_{n-1} f^{(n-1)}+\cdots+a_{1} f^{\prime}+a_{0} f=g
$$

are entire functions. Then all the solutions of (2.4) are entire functions.

Lemma 6 (see [1]). Let $h(z)$ be an entire function of order $\sigma(h)=\alpha<$ $1 / 2, A(r)=\inf _{|z|=r} \log |h(z)|$ and $B(r)=\sup _{|z|=r} \log |h(z)|$. If $\beta<\alpha<1$, then

$$
\underline{\log \operatorname{dens}}\{r: A(r)>\cos (\pi \alpha) B(r)\} \geq 1-\beta / \alpha .
$$

REMARK 2. In Lemma 6 , the lower logarithmic density of a set $E$ is defined by

$$
\underline{\log \operatorname{dens}} E=\liminf _{r \rightarrow \infty} \frac{\lambda(E \cap[1, r])}{\log r},
$$

where $\lambda(E \cap[1, r])$ is the logarithmic measure of $E \cap[1, r]$.

REMARK 3. By the definition of the logarithmic measure and logarithmic density of a set $E$, we know that if $\log \operatorname{dens} E>0$, then the logarithmic measure of $E$ is infinite.

Lemma 7. Let $f, a$ be two entire functions with $\sigma(a)=\sigma(f)$ and $\left\{z_{r}\right\}$ be a sequence of points such that $\left|z_{r}\right|=r$ and $\left|f\left(z_{r}\right)\right|=M(r, f)$. Then

$$
0 \leq \lim _{r \rightarrow \infty}\left|\frac{a\left(z_{r}\right)}{f\left(z_{r}\right)}\right| \leq A,
$$

where $A$ is a finite positive number.

Proof. Suppose that $\lim _{r \rightarrow \infty}\left|a\left(z_{r}\right) / f\left(z_{r}\right)\right|=\infty$. Then, for any positive number $B$, there exists $r_{0}$ such that

$$
\frac{\left|a\left(z_{r}\right)\right|}{M(r, f)}=\left|\frac{a\left(z_{r}\right)}{f\left(z_{r}\right)}\right|>B
$$

for $\left|z_{r}\right|=r>r_{0}$. From (2.8) we have

$$
B M(r, f)<\left|a\left(z_{r}\right)\right| \leq M(r, a)
$$

for $\left|z_{r}\right|=r>r_{0}$. By Definition 1 and (2.9), we have $\sigma(f)<\sigma(a)$, a contradiction. This completes the proof.

REMARK 4. The following example shows that $\lim _{r \rightarrow \infty}\left|a\left(z_{r}\right) / f\left(z_{r}\right)\right|$ can be zero in Lemma 7.

ExAmple. Let $f(z)=e^{z}$ and $a(z)=e^{-z}$. Obviously, $f(z)$ gets the maximum modulus and $a(z)$ gets the minimum modulus on the circle $|z|=r$ when $z \in \mathbb{R}^{+}$. Thus, we have $\lim _{r_{n} \rightarrow \infty}\left|a\left(z_{r_{n}}\right) / f\left(z_{r_{n}}\right)\right|=0$ for the sequence $\left\{z_{r_{n}}\right\} \subset \mathbb{R}^{+}$. 


\section{Proofs of theorems}

Proof of Theorem 1. By Lemma 5, $f$ is an entire function. Suppose that $f(z)$ is a nonconstant polynomial. Then from (1.1) we have

$$
a=\frac{f^{(k)}-e^{Q} f}{1-e^{Q}} .
$$

Hence $\sigma(a) \leq \operatorname{deg} Q$, which contradicts the hypothesis. Next we suppose that $f$ is a transcendental entire function. We discuss the following two cases.

CASE 1. Suppose that $e^{Q}$ is a constant, say $c \neq 0$. Then (1.1) can be rewritten as

$$
f^{(k)}-a=c(f-a) .
$$

If $\sigma(f)<\infty$, then $\mu_{2}(f)=\sigma_{2}(f)=\operatorname{deg} Q=0$, which yields the conclusion of Theorem 1.

Next we suppose that $\sigma(f)=\infty$. Then

$$
M(r, f) \rightarrow \infty \quad \text { as } r \rightarrow \infty .
$$

Let $M(r, f)=\left|f\left(z_{r}\right)\right|$, where $z_{r}=r e^{i \theta(r)}, \theta(r) \in[0,2 \pi)$. From (3.3) and Wiman-Valiron theory (see [9]), there exists a subset $F \subset(1, \infty)$ with finite logarithmic measure such that for some $z_{r}$ satisfying $\left|z_{r}\right|=r \notin F$ and $M(r, f)=\left|f\left(z_{r}\right)\right|$, we have

$$
\frac{f^{(k)}\left(z_{r}\right)}{f\left(z_{r}\right)}=\left(\frac{\nu(r, f)}{z_{r}}\right)^{k}(1+o(1))
$$

as $r(\notin F) \rightarrow \infty$. From the condition $\sigma(a)<\infty$ and Definition 1, we see that there exists an infinite sequence $z_{r_{n}}$ such that

$$
\lim _{r_{n} \rightarrow \infty} \frac{\log \log M\left(r_{n}, f\right)}{\log r_{n}}=\limsup _{r_{n} \rightarrow \infty} \frac{\log \log M\left(r_{n}, f\right)}{\log r_{n}}=\infty
$$

and

$$
\lim _{r_{n} \rightarrow \infty}\left|\frac{a\left(z_{r_{n}}\right)}{f\left(z_{r_{n}}\right)}\right|=\lim _{r_{n} \rightarrow \infty} \frac{\left|a\left(z_{r_{n}}\right)\right|}{M\left(r_{n}, f\right)}=0 .
$$

Since (3.2) can be rewritten as

$$
c=\frac{f^{(k)} / f-a / f}{1-a / f},
$$

from (3.4)-(3.7) we have

$$
c=\left(\frac{\nu\left(r_{n}, f\right)}{z_{r_{n}}}\right)^{k}(1+o(1))
$$

as $r_{n}(\notin F) \rightarrow \infty$. Proceeding as in the proof of Lemma 2.5 in [13] and 
applying (3.5), we get

$$
\lim _{r_{n} \rightarrow \infty} \frac{\log \log M\left(r_{n}, f\right)}{\log r_{n}}=\lim _{r_{n} \rightarrow \infty} \frac{\log \nu\left(r_{n}, f\right)}{\log r_{n}}=\infty,
$$

which contradicts (3.8).

CASE 2. Suppose that $e^{Q}$ is a nonconstant entire function. Then $\sigma\left(e^{Q}\right)=$ $\operatorname{deg} Q \geq 1$. We discuss the following two subcases:

SubCASE 2.1. Suppose that $\sigma(f)=\infty$. Then we have

$$
\sigma(f)=\limsup _{r \rightarrow \infty} \frac{\log \nu(r, f)}{\log r}=\infty \text {. }
$$

Let

$$
Q:=q_{n} z^{n}+q_{n-1} z^{n-1}+\cdots+q_{1} z+q_{0},
$$

where $q_{n}(\neq 0), q_{n-1}, \ldots, q_{1}, q_{0}$ are complex numbers.

From (3.11) we get $\lim _{|z| \rightarrow \infty}\left|Q /\left(q_{n} z^{n}\right)\right|=1$. Hence there exists $r_{0}>0$ such that $\left|Q /\left(q_{n} z^{n}\right)\right|>1 / e$ for $|z|>r_{0}$. Combining this with (1.1) we get

$$
\begin{aligned}
n \log r+\log \left|q_{n}\right|-1 & <\log \left|\log e^{Q}\right| \leq\left|\log \log e^{Q}\right| \\
& =\left|\log \log \frac{f^{(k)}-a}{f-a}\right|=\left|\log \log \frac{f^{(k)} / f-a / f}{1-a / f}\right|
\end{aligned}
$$

when $|z|>r_{0}$. Since $\sigma(a)<\infty$ and $\sigma(f)=\infty$, from (3.4) and (3.5) we get

$$
\lim _{r_{n} \rightarrow \infty}\left|\frac{a\left(z_{r_{n}}\right)}{f\left(z_{r_{n}}\right)}\right|=\lim _{r_{n} \rightarrow \infty} \frac{\left|a\left(z_{r_{n}}\right)\right|}{M\left(r_{n}, f\right)}=0 .
$$

By substituting (3.4) and (3.13) into (3.12) we have

$$
n \log \left|z_{r_{n}}\right|+\log \left|q_{n}\right|-1 \leq\left|\log \log \left(\frac{\nu\left(r_{n}, f\right)}{z_{r_{n}}}\right)^{k}(1+o(1))\right|,
$$

as $\left|z_{r_{n}}\right|=r_{n}\left(>r_{0}\right) \rightarrow \infty, r_{n} \notin F$. Since

$$
\begin{aligned}
& \log \left(\frac{\nu\left(r_{n}, f\right)}{z_{r_{n}}}\right)^{k}(1+o(1)) \\
& \quad=k\left(1-\frac{\log r_{n}}{\log \nu\left(r_{n}, f\right)}-\frac{i \theta\left(r_{n}\right)}{\log \nu\left(r_{n}, f\right)}\right) \log \nu\left(r_{n}, f\right)+o(1)
\end{aligned}
$$

as $\left|z_{r_{n}}\right|=r_{n} \rightarrow \infty, r_{n} \notin F$, from (3.4), (3.5), Lemma 3 and the condition $\theta\left(r_{n}\right) \in[0,2 \pi)$ we get

$$
\begin{aligned}
n & \leq \limsup _{r \rightarrow \infty} \frac{\left|\log \log \left(\nu(r, f) / z_{r}\right)^{k}(1+o(1))\right|}{\log r} \\
& \leq \limsup _{r \rightarrow \infty} \frac{\log \log \nu(r, f)}{\log r}=\sigma_{2}(f) .
\end{aligned}
$$

From (3.11) we have $\sigma\left(e^{Q}\right)=\operatorname{deg} Q=n$. Thus, $\sigma\left(e^{Q}\right)=n \leq \sigma_{2}(f)$. 
On the other hand, from (1.1), we have

$$
|Q(z)|=\left|\log e^{Q}\right|=\left|\log \frac{f^{(k)} / f-a / f}{1-a / f}\right| .
$$

Substituting (3.4), (3.5) and (3.13) into (3.17) we get

$$
e^{Q}=\left(\frac{\nu\left(r_{n}, f\right)}{z_{r_{n}}}\right)^{k}(1+o(1))
$$

as $\left|z_{r_{n}}\right|=r_{n} \rightarrow \infty, r_{n} \notin F$. From (3.18) we get

$$
\left|Q\left(z_{r_{n}}\right)\right|=k\left|\log \nu\left(r_{n}, f\right)-\log r_{n}-i \theta\left(r_{n}\right)\right|(1+o(1))
$$

as $\left|z_{r_{n}}\right|=r_{n} \rightarrow \infty, r_{n} \notin F$. By (3.18), we have

$$
\limsup _{r_{n} \rightarrow \infty} \frac{\log \log \left(\frac{\nu\left(r_{n}, f\right)}{\left|z_{r_{n}}\right|}\right)^{k}(1+o(1))}{\log r_{n}} \leq \limsup _{r_{n} \rightarrow \infty} \frac{\log \log M\left(r_{n}, e^{Q}\right)}{\log r_{n}} .
$$

Since

$$
\limsup _{r_{n} \rightarrow \infty} \frac{\log \log \nu\left(r_{n}, f\right)}{\log r_{n}}=\limsup _{r_{n} \rightarrow \infty} \frac{\log \log \left(\nu\left(r_{n}, f\right)^{k} /\left|z_{r_{n}}\right|^{k}\right)}{\log r_{n}}
$$

and

$$
\limsup _{r_{n} \rightarrow \infty} \frac{\log \log \left(\nu\left(r_{n}, f\right)^{k} / 2 r_{n}^{k}\right)}{\log r_{n}} \leq \limsup _{r_{n} \rightarrow \infty} \frac{\log \log \left(\nu\left(r_{n}, f\right) /\left|z_{r_{n}}\right|\right)^{k}(1+o(1))}{\log r_{n}} .
$$

From (3.20)-(3.22) and Lemma 3, we get

$$
\sigma_{2}(f) \leq \sigma\left(e^{Q}\right)=n .
$$

Combining (3.23) with (3.16), we have $\sigma_{2}(f)=\operatorname{deg} Q=n$.

Additionally, from (3.12), (3.18) and the conditions $z_{r}=r e^{i \theta(r)}, \theta(r) \in$ $[0,2 \pi),\left|z_{r}\right|=r$, we get

$$
\begin{aligned}
n \log \left|z_{r}\right|+\log \left|q_{n}\right|-1 & \leq \log \left|Q\left(z_{r}\right)\right| \\
& \leq\left|\log \log e^{Q\left(z_{r}\right)}\right| \quad\left(r>r_{0}\right)
\end{aligned}
$$

and

$$
\begin{aligned}
\log e^{Q} & =k(\log \nu(r, f)-\log r-i \theta(r)+o(1)) \\
& =k(\log \nu(r, f)-\log r)(1+o(1))
\end{aligned}
$$

as $r \rightarrow \infty, r \notin F$. From (3.24), (3.25) and Lemma 4, we get

$$
n \leq \liminf _{r \rightarrow \infty} \frac{\log \log \nu(r, f)}{\log r}=\mu_{2}(f)
$$


Since $\mu_{2}(f) \leq \sigma_{2}(f)$, we have $\mu_{2}(f)=\sigma_{2}(f)=\operatorname{deg} Q=n$. Thus, $f$ satisfies our conclusion.

SubCASE 2.2. Suppose that $\sigma(f)<\infty$.

If $\sigma(f)>\sigma(a)$, from (3.6) we get

$$
\lim _{r_{n} \rightarrow \infty}\left|\frac{a\left(z_{r_{n}}\right)}{f\left(z_{r_{n}}\right)}\right|=\lim _{r_{n} \rightarrow \infty} \frac{\left|a\left(z_{r_{n}}\right)\right|}{M\left(r_{n}, f\right)}=0 .
$$

By a similar argument to Subcase 2.1, we get $n \leq \sigma_{2}(f)=0$ (see (3.16)). Since $Q$ is a nonconstant polynomial, we have $n \geq 1$. We get a contradiction.

If $\sigma(f)<\sigma(a)$, from (3.1) we get $\sigma(a) \leq \max \left\{\sigma(f), \sigma\left(e^{Q}\right)\right\}$. This contradicts our hypothesis.

If $\sigma(f)=\sigma(a)$, by Lemma 7 we have

$$
0 \leq \lim _{r \rightarrow \infty}\left|\frac{a\left(z_{r}\right)}{f\left(z_{r}\right)}\right| \leq A
$$

for any sequence $\left\{z_{r}\right\}$, where $A$ is a positive number.

Suppose that $\lim _{r \rightarrow \infty}\left|a\left(z_{r}\right) / f\left(z_{r}\right)\right| \neq 1$ for some sequence $\left\{z_{r}\right\}$. By (3.12)-(3.16) and (3.28) we have $n \leq \sigma_{2}(f)=0$. Since $Q$ is a nonconstant polynomial, we have $n \geq 1$, a contradiction.

Suppose now that $\lim _{r \rightarrow \infty}\left|a\left(z_{r}\right) / f\left(z_{r}\right)\right|=1$ for some sequence $\left\{z_{r}\right\}$. Equation (1.1) can be rewritten as

$$
f^{(k)}-e^{Q} f=a\left(1-e^{Q}\right) .
$$

Thus,

$$
\frac{f^{(k)}}{f}-e^{Q}=\frac{a}{f}\left(1-e^{Q}\right) .
$$

So, we have

$$
\left|e^{Q}\right|<\left|\frac{a}{f}\right|\left|1-e^{Q}\right|+\left|\frac{f^{(k)}}{f}\right|+O(1) .
$$

Since the order of $f$ is finite, by (3.4), Lemma 3 and $\lim _{r \rightarrow \infty}\left|a\left(z_{r}\right) / f\left(z_{r}\right)\right|$ $=1$, we get

$$
\frac{\log \log \left|e^{Q}\right|}{\log r}<\frac{\log \log \left|e^{Q}\right|}{\log r}
$$

for the sequence $\left\{z_{r}\right\}$ with $\left|z_{r}\right|=r(\notin F) \rightarrow \infty$, a contradiction.

Thus, the proof of Theorem 1 is complete.

Proof of Theorem 2. From Lemma 5, we know that $f$ is an entire function. Suppose that $\sigma_{2}(f)<\infty$. If $\sigma(f)<\infty$, from (1.3) we have $\sigma\left(e^{Q}\right) \leq$ $\max \{\sigma(f), \sigma(a)\}<\infty$. Since $Q(z)$ is a transcendental entire function, we have $\sigma\left(e^{Q}\right)=\infty$, a contradiction. Hence $\sigma(f)=\infty$. As $f$ is a nonconstant 
entire function we have

$$
M(r, f) \rightarrow \infty \quad \text { as } r \rightarrow \infty .
$$

From (3.33) and Wiman-Valiron theory (see [9]), there exists a subset $F \subset(1, \infty)$ with finite logarithmic measure such that for some points $z_{r}$ satisfying $\left|z_{r}\right|=r \notin F$ and $M(r, f)=\left|f\left(z_{r}\right)\right|$, we have

$$
\frac{f^{(k)}\left(z_{r}\right)}{f\left(z_{r}\right)}=\left(\frac{\nu(r, f)}{z_{r}}\right)^{k}(1+o(1))
$$

as $r(\notin F) \rightarrow \infty$. By the condition $\sigma(a)<\infty$ and Definition 1, there exists an infinite sequence $z_{r_{n}}$ such that

$$
\lim _{r_{n} \rightarrow \infty} \frac{\log \log M\left(r_{n}, f\right)}{\log r_{n}}=\limsup _{r_{n} \rightarrow \infty} \frac{\log \log M\left(r_{n}, f\right)}{\log r_{n}}=\infty
$$

and

$$
\lim _{r_{n} \rightarrow \infty}\left|\frac{a\left(z_{r_{n}}\right)}{f\left(z_{r_{n}}\right)}\right|=\lim _{r_{n} \rightarrow \infty} \frac{\left|a\left(z_{r_{n}}\right)\right|}{M\left(r_{n}, f\right)}=0 .
$$

From (1.3) and (3.33)-(3.36), we have

$$
e^{Q\left(z_{r_{n}}\right)}=\left(\frac{\nu\left(r_{n}\right)}{z_{r_{n}}}\right)^{k}(1+o(1))+o(1),
$$

where $\nu\left(r_{n}\right)$ is the central index of $f$. Since $\sigma(f)=\infty$, Lemma 2 shows that $\nu\left(r_{n}\right)$ satisfies $\nu\left(r_{n}\right) \geq\left|z_{r_{n}}\right|^{N}$ for any sufficiently large positive number $N$, as $\left|z_{r_{n}}\right|=r_{n} \rightarrow \infty, r_{n} \notin F$. So we have

$$
\begin{aligned}
\left|Q\left(z_{r_{n}}\right)\right| & \leq|\log |\left(\frac{\nu\left(r_{n}\right)}{z_{r_{n}}}\right)^{k}(1+o(1))+o(1)||+2 \pi \\
& \leq k \log \nu\left(r_{n}\right)+o(1)
\end{aligned}
$$

as $\left|z_{r_{n}}\right|=r_{n} \rightarrow \infty, r_{n} \notin F$. By Lemma 3, we have

$$
\frac{\log \log \nu\left(r_{n}\right)}{\log r_{n}} \leq \sigma_{2}(f)+1
$$

for sufficiently large $r_{n}$. From (3.38) and (3.39), we have

$$
\left|Q\left(z_{r_{n}}\right)\right| \leq r^{\sigma_{2}(f)+1}+O(1)
$$

as $\left|z_{r_{n}}\right|=r_{n} \rightarrow \infty, r_{n} \notin F$. By Lemma 6 , there exists a set $H \subset(1, \infty)$ with infinite logarithmic measure such that

$$
\left|Q\left(z_{r_{n}}\right)\right| \geq M\left(r_{n}, Q\right)^{c}
$$

for $\left|z_{r_{n}}\right|=r_{n} \in H$, where $0<c<1$. From (3.40) and (3.41) we have 


$$
\frac{M(r, Q)^{c}}{r^{\sigma_{2}(f)+1}} \leq 1
$$

for $r_{n} \in H \backslash F$ and $\left|f\left(z_{r_{n}}\right)\right|=M\left(r_{n}, f\right)$. Since $Q$ is transcendental, we have

$$
\frac{M\left(r_{n}, Q\right)^{c}}{r_{n}^{\sigma_{2}(f)+1}} \rightarrow \infty
$$

as $r_{n} \rightarrow \infty$, a contradiction. Thus, the proof of Theorem 2 is complete.

Acknowledgments. The authors wish to express their thanks to the referee for his/her valuable suggestions and comments.

\section{References}

[1] P. D. Barry, On a theorem of Besicovitch, Quart. J. Math. Oxford 14 (1963), 293-302.

[2] R. Brück, On entire functions which share one value CM with their first derivative, Results Math. 30 (1996), 21-24.

[3] Z. X. Chen and C. C. Yang, Some further results on the zeros and growths of entire solutions of second order linear differential equations, Kodai Math. J. 22 (1999), 273-285.

[4] Z. X. Chen and Z. L. Zhang, Entire functions sharing fixed points with their higherorder derivatives, Acta Math. Sinica (Chinese Ser.) 50 (2007), 1213-1222.

[5] G. G. Gundersen and L. Z. Yang, Entire functions that share one value with one or two of their derivatives, J. Math. Anal. Appl. 223 (1998), 88-95.

[6] W. K. Hayman, Meromorphic Functions, Clarendon Press, Oxford, 1964.

[7] -, Slowly growing integral and subharmonic functions, Comment. Math. Helv. 34 (1960), 75-84.

[8] G. Jank und L. Volkmann, Einführung in die Theorie der ganzen und meromorphen Funktionen mit Anwendungen auf Differentialgleichungen, Birkhäuser, Basel, 1985.

[9] I. Laine, Nevanlinna Theory and Complex Differential Equations, de Gruyter, Berlin, 1993.

[10] X. M. Li and C. C. Gao, Entire functions sharing one polynomial with their derivatives, Proc. Indian Acad. Sci. Math. 118 (2008), 13-26.

[11] H. F. Liu and D. C. Sun, On the uniqueness problems of entire functions and their derivatives, J. Math. Anal. Appl. 348 (2008), 614-619.

[12] L. Rubel and C. C. Yang, Values shared by an entire function and its derivative, in: Complex Analysis (Lexington, KY, 1976), Lecture Notes in Math. 599, Springer, Berlin, 1977, 101-103.

[13] J. Wang and X. M. Li, The uniqueness of an entire function sharing a small entire function with its derivatives, J. Math. Anal. Appl. 354 (2008), 478-489.

[14] J. P. Wang, Entire functions that share a polynomial with one of their derivatives, Kodai Math. J. 27 (2004), 144-151.

[15] L. Yang, Value Distribution Theory and New Research, Science Press, Beijing, 1982 (in Chinese).

[16] L. Z. Yang, Solution of a differential equation and its applications, Kodai Math. J. 22 (1999), 458-464. 
[17] H. X. Yi and C. C. Yang, Uniqueness Theory of Meromorphic Functions, Science Press and Kluwer, Beijing, 2003.

Guowei Zhang, Ang Chen

Department of Mathematics

Shandong University

Jinan, Shandong 250100, P.R. China

E-mail: zhirobo@yahoo.com.cn

ang.chen.jr@gmail.com

Received 27.4.2009

and in final form 25.7.2009 